\title{
There's an App for That: A Guide for Healthcare Practitioners and Researchers on Smartphone Technology
}

\author{
Nasser F BinDhim ${ }^{1,2^{*}}$, Lyndal Trevena ${ }^{2}$ \\ 1. Health Informatics Department, College of health Sciences, Saudi Electronic University. Saudi \\ Arabia, Riyadh.
}

2. School of Public Health, University of Sydney, Australia, New South Wales.

\begin{abstract}
Smartphone technology is nascent compared to other technologies; however, it has shown an unprecedented uptake amongst lay consumers and professionals. This article presents the history, components, and key features of smartphones, as well as their related concepts and how they work, and it also delineates the process of smartphone applications (apps) development and publishing in the app stores. It also describes and discusses smartphone technology utilisation for health consumers, healthcare professionals, and health researchers, as well as the regulations of health-related apps.

Correspondence: nbin6641@uni.sydney.edu.au

DOI: 10.5210/ojphi.v7i2.5522

Copyright @2015 the author(s)

This is an Open Access article. Authors own copyright of their articles appearing in the Online Journal of Public Health Informatics. Readers may copy articles without permission of the copyright owner(s), as long as the author and OJPHI are acknowledged in the copy and the copy is used for educational, not-for-profit purposes
\end{abstract}

\section{WHAT IS A SMARTPHONE?}

There is no universally agreed-upon definition for a 'smartphone'. However, in one definition, a smartphone is considered 'a mobile phone handset with advanced hardware and software capabilities that enable it to perform complex functions similar to those of laptop computers' [1]. There are a few key components necessary for a mobile phone handset to be a smartphone: (a) It should be a novice appliance designed for personal use, rather than designed for business or commercial use. This requirement (being a novice appliance) helps exclude personal digital assistants (PDAs), which are often developed for business use and may sometimes be called smartphones [2]. (b) The second component is the ability to connect to the Internet in a constant, unrestricted way, which helps the user to exchange and generate data 'on the go'. (c) Third is the ability to install a range of applications (apps) from an external source, such as an 'app store'. (d) The fourth component (which is optional but increasingly common) is being large, with a high-resolution screen and a high-definition camera to facilitate high-quality video conferencing and advanced use for areas such as ultrasound and telemedicine.

\section{THE HISTORY OF SMARTPHONES}

In his article, 'The History of Smartphones: Timeline', Arthur (2012) states that the timeline of smartphones began in January 2007, when the Apple iPhone was revealed for the first time [3]. Kim (2011) more accurately divided the history of the smartphone into two main eras: (1) pre-iPhone and (2) post-iPhone [2]. 
Smartphones in the pre-iPhone era were largely conceptualised as office machines and seen as enhanced PDAs, with features like the ability to make phone calls, address books, calculators, calendars, and the abilities to send e-mail and faxes [2]. These features usually stood alone and could not communicate with each other. An example of a successful preiPhone device was the Blackberry, which was introduced in 2002 and incorporated a range of office functions, such as Internet and fax functions [2]. Another pre-iPhone operating system (OS) was the Windows Pocket PC 2000, which later evolved into Windows Mobile 5 and 6 [4]. Thus, smartphones during this era could be summarised as mobile phones with extra advancements that usually came as a package of apps decided by the manufacturer, regardless of the user's needs, and targeted to professionals, rather than the general population.

On the other hand, smartphones in the post-iPhone era are widely seen as personal computers with mobile-phone functions. They have transformed from mainly data receivers to data generators, and there is no limit to their capabilities to assist in any situation. Smartphones have replaced many things in our lives-people no longer need maps for a new city or timetables for public transport [5]. Smartphones can store people's 'to-do' lists amongst all of their organising functions and alarms, as well as gym programs, diet plans, and medication reminders - the list goes on and on [5]. However, unlike the pre-iPhone era, the range and types of apps on the devices are decided by the users, based on their own needs. Moreover, post-iPhone apps are able to communicate and share data between each other. For example, apps can access contact lists or images taken by the built-in camera and transfer them to another app for processing or utilise them to perform a new function.

Eventually, the success of the post-iPhone OSs has forced the leading pre-iPhone OS Blackberry to consider stopping production [6,7]. Windows Mobile was also renovated to cope with the advances of the post-iPhone era [8].

\section{KEY FEATURES OF SMARTPHONE APPS}

Smartphone users can download apps from app stores using the advanced functionalities of smartphones and tablets [9]. These apps are comparable to those run on a personal computer (PC), which enables smartphones to replace laptops or PCs in most tasks, such as Web browsing, document processing, video and music playing, task managing, and video game playing [9].

Portable accessibility: The main advantage of smartphone apps over previous computer technology is its portability. We can claim that any health-related Internet website is accessible anywhere and at any time, but the fact is that it is only accessible wherever one has the hardware and an Internet connection. In contrast, as users carry their smartphones with them all the time, they are available whenever they need them. This saves time and potentially offers more privacy and anonymity. Such proximity to the consumer gives smartphones great potential as a health promotion tool [10]. According to a recent Google study, 51\% of smartphone users search the Internet 'on the go' with their device [11]. They are used while multi-tasking with other media (e.g., while watching TV, using the Internet on a computer, and listening to music) [12].

Storage: Apps can store user inputs, organise them, and generate new data that can be stored on the device and retrieved via the app. Also, most of the apps' multimedia content is stored locally and can be accessed anytime and anywhere. Apps also have the ability to download more content when an Internet connection is available, and in addition to local storage apps can store data on remote Web servers for the user to access via other devices or computers. Apps can be easily updated with new data downloaded directly onto the app, or the user can update the app via the app store with several clicks. 
Notifications: Some of the most novel features of smartphone apps are local notifications and push notifications. Notifications are a short message service (SMS)-like function that is free of cost and more interactive. According to Apple's technical description, notifications ensure immediate, time-sensitive delivery, even when an app is not running [13]. Local notifications are generated from the app itself, usually as reminders or an alarm clock, and are repeatable [13]. However, push notifications are server-generated and can initiate an interactive process inside the app [13]. Push notifications use a unique device identification (which is different for each app) that acts as a phone number, so the server can deliver the push-notification message to a specific device that uses a specific app [13]. Users can enable or disable the notification feature for a specific app, which gives users the ability to control which apps they want to receive notifications from. In addition, the remote server can also receive information about whether users have enabled the notification function. This feature gives the notification services superior spam control over those of SMS.

Ad-hoc devices: An 'ad-hoc device' is an add-on developed to extend the utilisation of the main device in an unintended way. For example, a smartphone is not intended to be used as a medical device; however, ad-hocs can be developed to extend its utilisation as a medical device. Smartphone apps can be built to interrupt data from ad-hoc devices, which extend the potential utilisation of apps to an unprecedented level. There are currently various medical ad-hoc devices available for health consumers and healthcare professionals, including blood pressure, heart rate, blood glucose level, and ultrasound scanners [14].

\section{KEY FEATURES OF APP STORES}

App stores are global, virtual stores that sell apps, books, music, and movies. Their power comes from the fact that, although users can modify their phones to download apps from other sources, each app store is the only marketplace for selling and buying apps for a specific smartphone OS. For example, the Apple App Store is the only marketplace where iPhone, iPod, and iPad users can get apps for their devices. The App Store also comes with the default apps package in these devices.

The two largest app stores are Apple's App Store and Google Play (previously called the Android Market), which is the main source of apps for the Android OS. The latter is now used by many mobile phone handset manufacturers, such as HTC, Motorola, and Samsung. In 2009, after nine months in business, App Store consumers had downloaded one billion apps [15]. In 2013, Apple users had downloaded 50 billion apps, up from 15 billion in 2011 $[16,17]$. Android users had also downloaded about 48 billion apps by 2013, up from 10 billion in 2011 [18,19].

How they work: App Stores offer developer subscriptions, which are accounts for app publishers allowing them to publish their apps on the app stores [20,21]. If an app publisher decides to sell an app, a percentage of the app's cost will be given to the app stores [20,21].

A publisher that is ready to submit an application has to provide information about the app, including the app's name, a description to be used in the advertisement, a price (if not free), and the category in which the publisher wants the app to be published [1]. In the Apple App Store, the publisher must also provide keywords for users to locate their apps. Google Play, on the other hand, searches the app description, instead of using keywords. Thus, in both stores, developers can intentionally select their keywords so that a user can find his or her app in addition to browsing by category.

The publisher can specify the countries in which the app is to be published and can customize the app for a specific language [22,23]. However, the ability to limit an app to a specific 
country is not entirely accurate. In a previous feasibility study, some users from countries that were not included in an app store's country list were able to download the study app from both the Apple and Google Play stores [10].

Finally, only in Apple app store the apps go through a review process - which takes between 1 and 2 weeks, on average-where an app store reviewer will perform a technical compatibility and content verification review [24].

App Ranking and Exposure: App stores rank the apps based mainly on the number of downloads. App publishers consider their app rank to reflect the number of app downloads [25]. There are now companies that aggregate the daily app rankings data from app stores. For example, Appannie [26] allows users to search for an individual app's current ranking, its lifetime ranking, and the countries and categories in which it has been highly ranked [25]. Distimo, an app-tracking company, monitors daily rankings in the app stores on a country-bycountry basis. It found that, to rank amongst the top 10-25 in the USA, an app needed between 45,000 and 80,000 downloads per day [25,27,28]. To rank amongst the top 10 to 25 in Australia, the UK, Germany, France, or Italy, an app only requires 4,000 to 18,000 daily downloads $[25,27,28]$. In most countries, an app needs approximately 3,000 daily downloads to achieve a top 50 ranking $[25,27,28]$. Although app rankings are mostly influenced by the number of downloads, some app publishers also believe that app reviews contribute to improving the ranking. Consequently, many app review companies now provide massive review services to app publishers - they can write positive reviews with 5-star ratings for the publisher's app or give bad reviews and ratings to competitors' apps [29,30].

The main criticism of the app ranking process is that the high-ranking apps will get more exposure and downloads and stay at the top of the list, thereby reducing the exposure of new apps. However, app stores also have ranked lists of newly released apps based on the date of release, so that they, too, can get more exposure. App stores can also feature an app by listing it on a special list or improve its ranking in a specific country. However, the criteria for featuring apps are unclear.

Are app stores the only way to access apps? For both Google Play and the Apple App Store, publishers need to release their app in the store if they want it to be downloaded by the app store's users. However, an app that is intended for a specific group of users (e.g., an organisation's staff or study participants) can be installed onto the users' devices directly from a website or the developer computer without the need to release it to the app store.

\section{SMARTPHONES AND HEALTH}

For health consumers: There are growing trends of health apps targeting health consumers. Such apps are usually released in the Health and Fitness category of app stores. Health apps have the potential to transform healthcare and health promotion in the community [31]. The number of health-related apps on the Apple App Store reached 13,000 apps by 2012 [32]. Many studies have identified health-related apps targeting consumers in the app stores. The apps cover many topics, including smoking cessation [33], ophthalmology [34], hernias [35], diabetes, obesity and weight management [36], asthma [37], personal health records [38], HIV and other sexually transmitted diseases [39], colorectal diseases [40], cancer [41], palliative care [42], pain management [43], fitness and physical activity [44], medication management and adherence [45], women's health, and child care [46], and depression [47]. However, although hundreds of health apps target consumers, very few are evidence-based, and many are with low-quality content $[37,41,43]$. 
In Australia, 28\% of smartphone users had used a health-related smartphone app, and 13\% used their smartphone for health-related activates at least once a week [48]. In 2012, the Pew Internet Research Center found that $53 \%$ of adults in the United States (US) owned a smartphone, $31 \%$ of whom had used their smartphone to search for health information and 19\% had downloaded and used an app to manage and monitor their health [49]. Furthermore, health-related apps can efficiently reach people in multiple countries who are searching for assistance to change health behaviours, such as giving up smoking [10]. In addition, healthrelated apps appear to be independent and perceived to be useful, regardless of e-health literacy and health information orientation [50].

Finally, In May 2014, Apple announced its Health app and HealthKit for developers. The Health app (Figure 1) records diagnostics, fitness, lab results, medications, nutrition, and vital signs [51]. The app shares data with other health apps in two directions and collects data from other health apps used by the user. This step by Apple suggests that health apps are growing in importance and have the potential to change the healthcare landscape. In addition, including health-related apps with smartphones will increase the awareness of and exposure to these apps and eventually increase their use, as more users will become interested in such apps after recognising their abilities.

Figure 1: Screenshots of the Apple ‘Health’ app to be released September 2014.

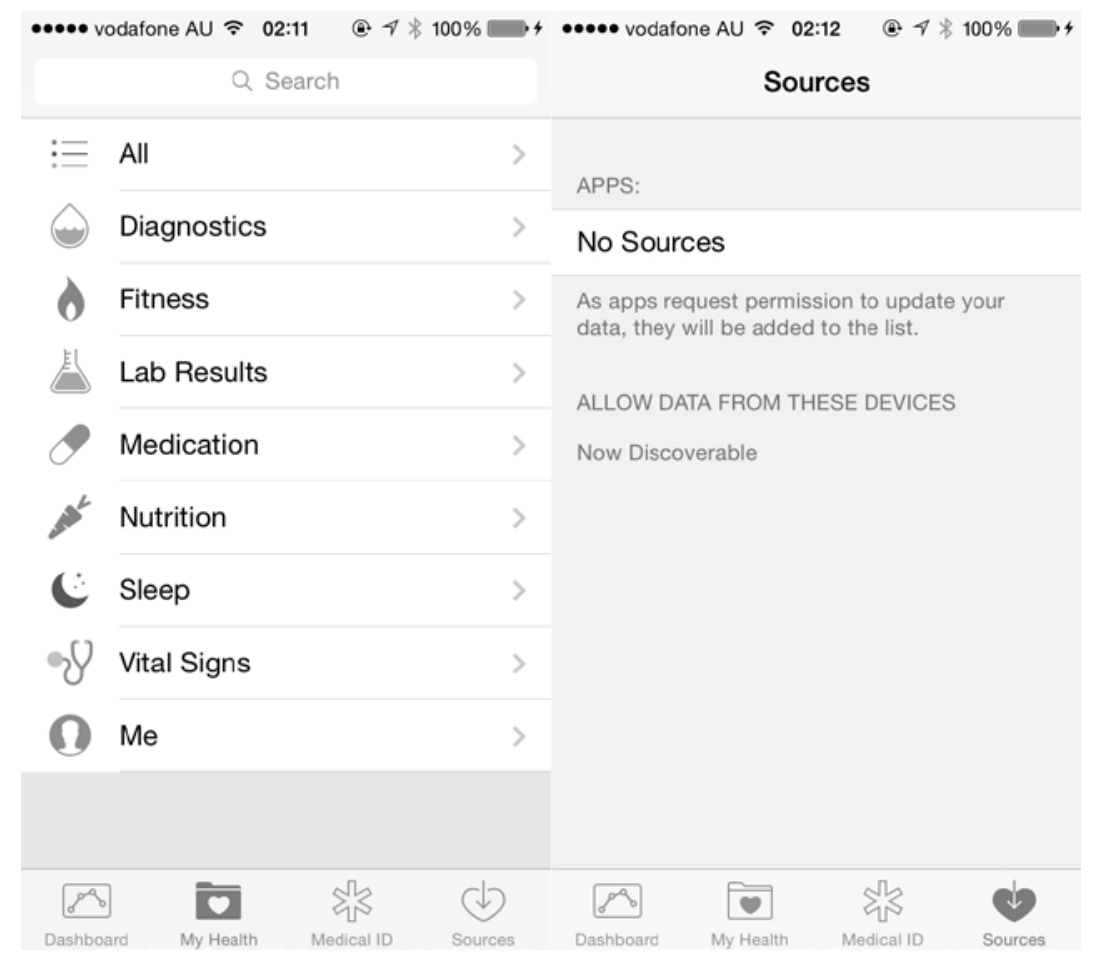

For health professionals: There are also growing numbers of apps targeting health consumers that are usually released in the Medical or References category of app stores. Those apps mostly claim to be developed to assist healthcare professionals in their daily tasks. At the same time, the use of smartphones by healthcare professionals is also increasing. In 2011, 75\% of physicians in the US used iPads or iPhones [52]. Another study in the US also found that $89 \%$ of residents and $98 \%$ of faculty staff owned smartphones, with $57 \%$ of house staff and $28 \%$ of attending physicians reporting regular personal use of smartphones during attending rounds [53]. Other healthcare professionals also use smartphone apps as references or point-of-care tools, including pharmacists [54,55], nurses [56], and dieticians [57]. In addition, smartphone apps are also used by medical students [58] and for medical 
training [59]. Apps targeting various healthcare professionals are also available in the app stores [55,60,61]. However, although many support the use of smartphones by healthcare professionals, which has the potential to improve patient care, the possible dangers associated with their use (e.g., breaches of patient confidentiality, conflicts of interests, and malfunctioning clinical decision-making apps) need to be investigated [62]. Finally, Apple has recently announced a partnership with Mayo Clinic to bring new innovations to patient care and improve the quality of health apps by developing new apps targeting patients and health professionals [63].

For health researchers: The proximity to the user, great computational capabilities, and storage make smartphone apps ideal research tools that are capable of delivering complex interventions. Apps can collect, store, and transfer data to a remote research database or export data directly to a local computer. In addition, research participants can be recruited directly from app stores [10]. Various studies have explored the use of smartphone apps for health research, including cross-sectional [10], observational [64], and randomised controlled trial [65]. In addition, smartphone apps have been used to conduct cross-country studies $[10,65]$.

However, few studies have been conducted to investigate the efficacy of smartphone app interventions. Of those studies, one found that a smartphone medication self-management app improved adherence and helped reduce rates of forgetting and of medication errors in elderly patients [66]. Moreover, a weight-loss pilot study found that the trial retention was 93\% at 6 months in the smartphone group, compared to $55 \%$ in the website group and $53 \%$ in the paper diary group [67].

Health-related smartphone apps are often hastily developed and may not meet consumers' or healthcare professionals' needs or expectations [68,69]. Recent studies have searched app stores for specific health-related apps and reviewed their quality [34,37,43,70], finding that there were methodological and quality problems that are common amongst health apps [68]. Moreover, other studies have found apps that encourage harmful health behaviours [1,9,25].

Thus, health-related research using smartphone apps is likely to rapidly evolve in the years to come. Therefore, more research efforts are needed to harness the potential of smartphone technology to improve healthcare.

Regulation of health apps: The US Food and Drug Administration (FDA) has focused on a small subset of mobile apps that meet the regulatory definition of 'devices' and that (1) are intended to be used as an accessory for a regulated medical device or (2) transform a mobile platform into a regulated medical device [71]. The FDA has also stated that, although many mobile apps carry minimal risks, those that can pose a greater risk to patients will require FDA review [71]. In addition, the FDA recently released a memorandum of a meeting after discussing glucose monitoring sensors with Apple executives [72]. The FDA stated that it 'will regulate based on the intended use of a device. Using the glucometer example, the glucometer may be unregulated if the intent is for a user to follow their blood sugar for the purposes of better nutrition. If the glucometer is marketed for diabetics, however, it would more likely be regulated as a medical device' [72]. However, the lay users may not be able to know the difference between what is intended to be a medical device or not. Moreover the app stores currently contain many apps that target healthcare professionals, with functions such as medication dosage calculators, which may cause patients harm if they malfunction. Such apps are not currently regulated and seem to be ignored [73].

To conclude, although smartphone technology is nascent compared to other technologies, it has shown unprecedented uptake amongst lay consumers and professionals. Fast advances in 
apps and ad-hoc devices have increasing complexity, stability, and reliability. It has also shown a promising role in changing the future healthcare landscape through the increasing applications for health and perceived usefulness amongst health consumers and healthcare professionals.

\section{Contributors}

All authors made substantial contributions to editing and revising of the manuscript. NFB was responsible drafting of the manuscript. All authors read and approved the final manuscript.

No competing interests.

\section{Acknowledgment}

Thanks to (Mada H Basyouni) for her feedback and comments on the first draft.

\section{References}

1. BinDhim NF. 2014. Freeman B, Trevena L. 2014. Pro-smoking apps for smartphones: the latest vehicle for the tobacco industry? Tob Control. 23(1), e4. PubMed

2. Kim P. 2011. The Apple iPhone Shock in Korea. Inf Soc. 27(4), 261-68. http://dx.doi.org/10.1080/01972243.2011.583826

3. Arthur C. 2012. The History Of Smartphone Timeline: The Guardian; [cited 3]. 2012]. Available from: http://www.theguardian.com/technology/2012/jan/24/smartphonestimeline.

4. De Herrera C. Versions of Windows CE / Windows Mobile [03/06/2014]. Available from: http://www.pocketpcfaq.com/wce/versions.htm.

5. BinDhim NF. 2012. Smartphone Apps importance as a health promotional tools: Sydney Gulf Forum. Available from: http://shproject.net/index.php?option=com_content\&view=article\&id=8.

6. Sharp A, Leske N. 2014. BlackBerry may stop making cellphones: CEO. Available from: http://www.thebarrieexaminer.com/2014/04/09/blackberry-may-consider-exitinghandsets-eyes-investments.

7. Miller H. 2013. BlackBerry Allies Waver as Device Maker May Go Private [04/06/2014]. Available from: http://www.bloomberg.com/news/2013-09-26/blackberryallies-waver-as-smartphone-maker-seeks-to-go-private.html.

8. Ballmer S, Belfiore J. 2012. Steve Ballmer and Joe Belfiore: Windows Phone 8 Launch Event. Available from: https://www.microsoft.com/en-us/news/speeches/2012/1029windowsphone8.aspx.

9. Bindhim NF, Naicker S, Freeman B, McGeechan K, Trevena L. 2014. Apps Promoting Illicit Drugs-A Need for Tighter Regulation? J Consum Health Internet. 18(1), 31-43. http://dx.doi.org/10.1080/15398285.2014.869166

10. BinDhim FN, McGeechan K, Trevena L. 2014. Who Uses Smoking Cessation Apps? A Feasibility Study Across Three Countries via Smartphones. JMIR Mhealth Uhealth. February 06;2(1):e4.

11. Think with Google. 2014. Going Local: How Advertisers Can Extend Their Relevance With Search [03/06/2014]. Available from: 
http://www.thinkwithgoogle.com/articles/how-advertisers-can-extend-their-relevancewith-search.html.

12. Google mobile ads. 2012. New research shows 6 countries are the clear leaders in smartphone adoption. Do you know which ones? [04/03/2014]. Available from: http://googlemobileads.blogspot.com.au/2012/05/new-research-shows-6-countriesare.html.

13. Apple. Local and Push Notifications. Available from: https://developer.apple.com/notifications/.

14. Sutton M, Fraser M. 2013. The rise of smartphone health and medical apps: Life Scientist; [07/05/2014]. Available from: http://lifescientist.com.au/content/biotechnology/article/the-rise-of-smartphone-healthand-medical-apps-1072193834.

15. Pope S. 2009. Apple's revolutionary app store downloads top one billion in just nine months. Available from: http://www.apple.com/pr/library/2009/04/24ApplesRevolutionary-App-Store-Downloads-Top-One-Billion-in-Just-Nine-Months.html.

16. James J. 2013. Apple's App Store Marks Historic 50 Billionth Download. Available from: http://www.apple.com/au/pr/library/2013/05/16Apples-App-Store-Marks-Historic50-Billionth-Download.html.

17. Pope S. 2011. Apple's app store downloads top 15 billion Available from: http://www.apple.com/pr/library/2011/07/07Apples-App-Store-Downloads-Top-15Billion.html.

18. Arthur C. 2013. Apple and Google app downloads reveal rate of smartphone growth. Available from: http://www.theguardian.com/technology/2013/may/16/apple-googleapp-downloads-smartphone.

19. The official Google blog. 2011. 10 billion Android Market downloads and counting. Available from: http://googleblog.blogspot.com.au/2011/12/10-billion-android-marketdownloads-and.html.

20. Android Developer. 2008. Android Market: Now available for users [22/02/2013]. Available from: http://android-developers.blogspot.com.au/2008/10/android-marketnow-available-for-users.html.

21. iOS developer program. Distribute [23/02/2013]. Available from: https://developer.apple.com/programs/ios/distribute.html.

22. iOS Developer Library. Your First App Store Submission: Create Your App Record in iTunes Connect [22/02/2012]. Available from: https://developer.apple.com/library/ios/\#documentation/ToolsLanguages/Conceptual/Yo urFirstAppStoreSubmission/CreateYourAppRecordiniTunesConnect/CreateYourAppRec ordiniTunesConnect.html\#//apple_ref/doc/uid/TP40011375-CH6-SW1.

23. Android Market for Developer. Upload applications [22/02/2012]. Available from: http://support.google.com/androidmarket/developer/bin/answer.py?hl=en\&answer=1134 69.

24. iOS Developer Library. Your First App Store Submission: Next Steps [12/04/2014]. Available from: https://developer.apple.com/library/ios/\#documentation/ToolsLanguages/Conceptual/Yo 
urFirstAppStoreSubmission/NextSteps/NextSteps.html\#//apple_ref/doc/uid/TP40011375 -CH11-SW1.

25. BinDhim NF. 2013. Freeman B, Trevena L. 2015. Pro-smoking apps: where, how and who are most at risk. Tob Control. 24(2).159-161.

26. Appannie. About us [12/11/2012]. Available from: http://www.appannie.com/.

27. Spriensma GJ. 2012. Quora answering series: download volume needed to hit the top charts: Distimo; [08/10/2012]. Available from: http://www.distimo.com/blog/2011_12_quora-answering-series-download-volumeneeded-to-hit-the-top-charts/.

28. Spriensma GJ. 2012. Quora answering series: download volume needed to hit top 25 per category: Distimo; [08/10/2012]. Available from: http://www.distimo.com/blog/2012_05_quora-answering-series-download-volumeneeded-to-hit-top-25-per-category/.

29. App review services [08/03/2014]. Available from: http://www.appreviewservice.com/.

30. I will provide 12 reviews or 15 ratings to your app on android store for $\$ 5$ [10/03/2014]. Available from: http://www.fiverr.com/yashvikas351/give-15-feedback-on-reviews-toany-ios-app.

31. Terry M. 2010. Medical apps for smartphones. Telemed J E Health. 16(1), 17-22. PubMed http://dx.doi.org/10.1089/tmj.2010.9999

32. Dolan B. 2011. Report: 13K iPhone consumer health apps in 2012 [06/06/2014]. Available from: http://mobihealthnews.com/13368/report-13k-iphone-consumer-healthapps-in-2012/.

33. Abroms LC, Padmanabhan N, Thaweethai L, Phillips T. 2011. iPhone Apps for Smoking Cessation: A Content Analysis. Am J Prev Med. 40(3), 279-85. PubMed http://dx.doi.org/10.1016/j.amepre.2010.10.032

34. Chhablani J, Kaja S, Shah V. Smartphones in ophthalmology2012 March 1, 2012. 127-31 p.

35. Connor K, Brady RRW, Beaux A, Tulloh B. 2013. Contemporary hernia smartphone applications (apps) [English.]. Hernia. 18, 557-61. PubMed

36. Gan KO, Allman-Farinelli M. 2011. A scientific audit of smartphone applications for the management of obesity. Aust N Z J Public Health. 35(3), 293-94. PubMed http://dx.doi.org/10.1111/j.1753-6405.2011.00707.x

37. Huckvale K, Car M, Morrison C, Car J. 2012. Apps for asthma self-management: a systematic assessment of content and tools. BMC Medicine.10(1):144. PubMed PMID: doi:10.1186/1741-7015-10-144.

38. Kharrazi H, Chisholm R, VanNasdale D, Thompson B. 2012. Mobile personal health records: An evaluation of features and functionality. Int J Med Inform. 81(9), 579-93. PubMed http://dx.doi.org/10.1016/j.ijmedinf.2012.04.007

39. Muessig KE, Pike EC, LeGrand S, Hightow-Weidman LB. 2013. Mobile Phone Applications for the Care and Prevention of HIV and Other Sexually Transmitted Diseases: A Review. J Med Internet Res. 15(1). http://dx.doi.org/10.2196/jmir.2301 
40. O’Neill S, Brady RRW. 2012. Colorectal smartphone apps: opportunities and risks. Colorectal Dis. 14(9), e530-4. PubMed http://dx.doi.org/10.1111/j.14631318.2012.03088.x

41. Pandey A, Hasan S, Dubey D, Sarangi S. 2013. Smartphone Apps as a Source of Cancer Information: Changing Trends in Health Information-Seeking Behavior [English.]. $J$ Cancer Educ. 28(1), 138-42. PubMed http://dx.doi.org/10.1007/s13187-012-0446-9

42. Nwosu AC, Mason S. 2012. Palliative medicine and smartphones: an opportunity for innovation? BMJ Supportive \& Palliative Care. 2(1), 75-77. PubMed http://dx.doi.org/10.1136/bmjspcare-2011-000151

43. Rosser BA, Eccleston C. 2011. Smartphone applications for pain management. $J$ Telemed Telecare. 17(6), 308-12. PubMed http://dx.doi.org/10.1258/jtt.2011.101102

44. West JH, Hall PC, Hanson CL, Barnes MD, Giraud-Carrier C, Barrett J. 2011. There's an app for that: content analysis of paid health and fitness apps. Journal of medical Internet research.14(3):e72-e.

45. Dayer L, Heldenbrand S, Anderson P, Gubbins PO, Martin BC. 2013. Smartphone medication adherence apps: Potential benefits to patients and providers. Journal of the American Pharmacists Association: JAPhA. 53(2). http://dx.doi.org/10.1331/JAPhA.2013.12202

46. Robinson F, Jones C. 2014. Women's engagement with mobile device applications in pregnancy and childbirth. Pract Midwife. 17(1), 23-25. PubMed

47. BinDhim NF, Shaman A M, Trevena L, Basyouni MH, Pont LG, Alhawassi TM. 2015. Depression screening via a smartphone app: cross-country user characteristics and feasibility. J Am Med Inform Assoc. 22(1), 29-34. PubMed

48. Mackay M. Australian mobile phone lifestyle index2013 19/08/2013. Available from: http://www.aimia.com.au/enews/2013/Events/AMPLI\%202013/Ampli\%202013\%20Rep ort_Final_October\%2024.pdf.

49. Fox S, Duggan M. 2012. Mobile health 2012 [06/06/2014]. Available from: http://community.g.pewinternet.com/ /media/Files/Reports/2012/PIP_MobileHealth201 2_FINAL.pdf.

50. Cho J, Park D, Lee HE. 2014. Cognitive Factors of Using Health Apps: Systematic Analysis of Relationships Among Health Consciousness, Health Information Orientation, eHealth Literacy, and Health App Use Efficacy. J Med Internet Res. 16(5). PubMed http://dx.doi.org/10.2196/jmir.3283

51. Apple. 2014. iOS 8 Preview: Health [11/06/2014]. Available from: https://www.apple.com/ios/ios8/health/.

52. Sandlot Solutions. 2011. 75\% of physicians use iPad or iPhone, survey shows [11/06/2014]. Available from: http://www.sandlotsolutions.com/knowledgeresources/industry-news-items/2011/05/09/75-physicians-use-ipad-or-iphone-surveyshows.

53. Katz-Sidlow RJ, Ludwig A, Miller S, Sidlow R. 2012. Smartphone use during inpatient attending rounds: Prevalence, patterns and potential for distraction. J Hosp Med. 7(8), 595-99. PubMed http://dx.doi.org/10.1002/jhm.1950

54. Felkey B, Fox B. 2002. Emerging technology at the point of care. Journal of the American Pharmacists Association: JAPhA. 43(5) (Suppl 1), S50-51. PubMed 
55. Haffey F, Brady RR, Maxwell S. 2014. Smartphone apps to support hospital prescribing and pharmacology education: a review of current provision. Br J Clin Pharmacol. 77(1), 31-38. PubMed http://dx.doi.org/10.1111/bcp.12112

56. Wentzel J, van Gemert-Pijnen J, eds. Antibiotic Information App for Nurses. eTELEMED 2014, The Sixth International Conference on eHealth, Telemedicine, and Social Medicine; 2014.

57. Lieffers JR, Vance VA, Hanning RM. 2014. Use of Mobile Device Applications in Canadian Dietetic Practice. Can J Diet Pract Res. 75(1), 41-47. PubMed http://dx.doi.org/10.3148/75.1.2014.41

58. Robinson T, Cronin T, Ibrahim H, Jinks M, Molitor T, et al. 2013. Smartphone Use and Acceptability Among Clinical Medical Students: A Questionnaire-Based Study. J Med Syst. 37(3), 9936. PubMed http://dx.doi.org/10.1007/s10916-013-9936-5

59. Kalz M, Lenssen N, Felzen M, Rossaint R, Tabuenca B, et al. 2014. Smartphone Apps for Cardiopulmonary Resuscitation Training and Real Incident Support: A MixedMethods Evaluation Study. J Med Internet Res. 16(3). $\underline{\text { PubMed }}$ http://dx.doi.org/10.2196/jmir.2951

60. Aungst TD. 2013. Medical applications for pharmacists using mobile devices. Ann Pharmacother. 47(7-8), 1088-95. PubMed http://dx.doi.org/10.1345/aph.1S035

61. Franko OI. 2011. Smartphone apps for orthopaedic surgeons. Clin Orthop Relat Res. 469(7), 2042-48. PubMed http://dx.doi.org/10.1007/s11999-011-1904-0

62. Buijink AWG, Visser BJ, Marshall L. 2013. Medical apps for smartphones: lack of evidence undermines quality and safety. Evid Based Med. 18(3), 90-92. PubMed http://dx.doi.org/10.1136/eb-2012-100885

63. Apple. 2014. Mayo Clinic [cited 11/06/2014]. Available from: http://www.apple.com/uk/ipad/business/profiles/mayo-clinic/.

64. Patel V, Nowostawski M, Thomson G, Wilson N, Medlin H. 2013. Developing a smartphone 'app'for public health research: the example of measuring observed smoking in vehicles. $J$ Epidemiol Community Health. 67(5), 446-52. PubMed http://dx.doi.org/10.1136/jech-2012-201774

65. BinDhim FN, McGeechan K, Trevena L. 2014. Assessing the effect of an interactive decision-aid smartphone smoking cessation application (app) on quit rates: a doubleblind automated randomized control trial protocol. BMJ Open.

66. Mira JJ, Navarro I, Botella F, Borrás F, Nuño-Solinís R, Orozco D, et al. 2014. A Spanish Pillbox App for Elderly Patients Taking Multiple Medications: Randomized Controlled Trial. J Med Internet Res. April 04;16(4):e99.

67. Carter CM, Burley JV, Nykjaer C, Cade EJ. 2013. Adherence to a Smartphone Application for Weight Loss Compared to Website and Paper Diary: Pilot Randomized Controlled Trial. J Med Internet Res. April 15;15(4):e32.

68. BinDhim FN, Hawkey A, Trevena L. 2015. A systematic review of quality assessment methods for smartphone health apps. Telemed J E Health. 21(2), 97-104. PubMed http://dx.doi.org/10.1089/tmj.2014.0088

69. Doarn CR, Merrell RC. 2013. There's an App for That. Telemed J E Health. 19(11), 81112. PubMed http://dx.doi.org/10.1089/tmj.2013.9983 
70. Demidowich AP, Lu K, Tamler R, Bloomgarden Z. 2012. An evaluation of diabetes selfmanagement applications for Android smartphones. J Telemed Telecare. 18(4), 235-38. PubMed http://dx.doi.org/10.1258/jtt.2012.111002

71. Mobile Medical Applications FDA. [13/06/2014]. Available from: http://www.fda.gov/MedicalDevices/ProductsandMedicalProcedures/ConnectedHealth/ MobileMedicalApplications/default.htm?utm_source=twitterfeed\&utm_medium=twitter \#b.

72. Kurt S. 2014. Apple and the FDA have discussed FDA regulations regarding possible new mobile products, sensors and a glucometer [16/06/2014]. Available from: http://appletoolbox.com/2014/06/apple-fda-discussed-fda-regulations-regardingpossible-new-mobile-products-sensors-glucometer/.

73. BinDhim FN, Trevena L. (2015) Health-related smartphone apps: regulations, safety, privacy and quality. BMJ Innovation. 1;43-45. 